\title{
Quantum state selection, alignment, and orientation of large molecules using static electric and laser fields
}

\author{
Frank Filsinger, Jochen Küpper, ${ }^{*}$ and Gerard Meijer \\ Fritz-Haber-Institut der Max-Planck-Gesellschaft, Faradayweg 4-6, 14195 Berlin, Germany \\ Lotte Holmegaard $^{1}$, Jens H. Nielsen ${ }^{2}$, Iftach Nevo ${ }^{1}$, Jonas L. Hansen ${ }^{3}$, and Henrik Stapelfeldt ${ }^{1,3 \dagger}$ \\ ${ }^{1}$ Department of Chemistry, University of Aarhus, 8000 Aarhus C, Denmark \\ ${ }^{2}$ Department of Physics and Astronomy, University of Aarhus, 8000 Aarhus C, Denmark \\ ${ }^{3}$ Interdisciplinary Nanoscience Center (iNANO), University of Aarhus, 8000 Aarhus C, Denmark
}

(Dated: August 11, 2009)

\begin{abstract}
Supersonic beams of polar molecules are deflected using inhomogeneous electric fields. The quantumstate-selectivity of the deflection is used to spatially separate molecules according to their quantum state. A detailed analysis of the deflection and the obtained quantum-state selection is presented. The rotational temperatures of the molecular beams are determined from the spatial beam profiles and are all approximately $1 \mathrm{~K}$. Unprecedented degrees of laser-induced alignment $\left(\left\langle\cos ^{2} \theta_{2 D}\right\rangle=\right.$ $0.972)$ and orientation of iodobenzene molecules are demonstrated when the state-selected samples are used. Such state-selected and oriented molecules provide unique possibilities for many novel experiments in chemistry and physics.
\end{abstract}

PACS numbers: $37.20 .+\mathrm{j}, 33.15 .-\mathrm{e}$

\section{INTRODUCTION}

For a large range of experiments in chemistry and physics, a high level of control over the external and internal degrees of freedom of molecules is very beneficial. This includes control over the translational and the rotational motions, as well as the selection of a single quantum state or a small set of states. Such quantumstate-selected targets provide unique possibilities, for example, for manipulating the external degrees of freedom with static electric fields $[1,2]$ or optical fields $[3,4]$, or both $[5,6]$. The quantum-state selection also naturally discriminates between individual stereo-isomers of large molecules [7]. The resulting samples of aligned or oriented individual isomers offer unique prospects for novel experiments with complex molecules, such as femtosecond pump-probe measurements, x-ray or electron diffraction in the gas-phase [8,9], high-harmonic generation [10], or tomographic reconstructions of molecular orbitals [11]. Moreover, it would provide considerably increased control in reaction dynamics experiments [12].

Strong cooling can be achieved in supersonic expansions of molecules seeded in an inert atomic carrier gas. For small molecules (consisting of just a few atoms) only a few rotational states are populated at the typical temperatures on the order of $1 \mathrm{~K}$. For larger polyatomic systems rotational cooling down to or even below $1 \mathrm{~K}$ still leaves the molecular ensemble distributed over a considerable number of rotational states, thereby often

\footnotetext{
*Electronic address: jochen@fhi-berlin.mpg.de
}

${ }^{\dagger}$ Electronic address: henriks@chem.au.dk masking quantum state specific effects. State selection can be performed using inhomogeneous electric or magnetic fields. The possibility to deflect polar molecules in a molecular beam with an electric field was first described by Kallmann and Reiche in 1921 [13] and experimentally demonstrated by Wrede in 1927 [14]. As early as 1926, Stern suggested that the technique could be used for the quantum state separation of small diatomic molecules at low temperatures [15]. In 1939 Rabi introduced the molecular beam resonance method, by using two deflection elements of oppositely directed gradients in succession, to study the quantum structure of atoms and molecules [16]. Whereas deflection experiments allow the spatial dispersion of quantum states, they do not provide any focusing. For small molecules in low-fieldseeking states this issue could be resolved using multipole focusers with static electric fields. These were developed independently in 1954/55 in Bonn $[17,18]$ and in New York, where they were used to produce the population inversion for the first MASER experiments [19, 20]. About ten years later, molecular samples in a single rotational state were used for state specific inelastic scattering experiments by the Bonn group [21] and, shortly thereafter, for reactive scattering $[22,23]$. In the following decades, multipole focusers were extensively used to study steric effects in gas-phase reactive scattering experiments $[12,24]$. Also for the investigation of steric effects in gas-surface scattering [25] and photodissociation [26] experiments, the preparation of oriented samples of stateselected molecules using electrostatic focusers was essential. For about ten years, it is also possible to manipulate the speed of small molecules using switched inhomogeneous electric fields in the so-called Stark decelerator [27]. More recently, also its optical [28] and mag- 
netic [29] analogs have been demonstrated.

Obtaining similar control over large molecules is more difficult, because all low-lying quantum states are highfield seeking at the required electric field strengths. In order to confine these molecules, dynamic focusing schemes are necessary [30-32]. Dynamic focusing of large molecules has been demonstrated in the alternatinggradient (AG) deceleration of benzonitrile [33] and in the conformer selection of 3-aminophenol [7]. However, if focusing is not necessary, spatial dispersion of quantum states can still be achieved using static electric fields in a Stern-Gerlach-type deflector. This molecular beam deflection has been used extensively as a tool to determine dipole moments and polarizabilities of molecular systems ranging from diatomics [14] over clusters [34] to large biomolecules [35].

Recently, we have demonstrated the quantum-state selection of large molecules [36] following the original proposal of Stern [15]. Here, the details of the electrostatic deflection are presented. It is shown, how the rotational temperature of cold supersonic jets can be determined with high precision from deflection measurements and that indeed a small subset of quantum states can be addressed in deflected samples of large molecules. In particular, the ground state has the largest Stark shift and molecules residing in this state are deflected the most. Our goal is to isolate and use rotational ground state molecules, or at least samples of molecules in the few lowest lying states, as targets for various experiments. Since the deflection does not change the initial state distribution but merely disperses it, it is crucial that the population of ground state molecules in the molecular beam is initially as large as possible. Therefore, the rotational temperature of the molecular beam is made as low as possible using a high-pressure supersonic expansion [37]. It is shown how the resulting state-selected molecules can be used to improve one-dimensional (1D) laser-induced alignment $[4,38]$ and mixed field orientation $[36,39,40]$. Here, alignment refers to confinement of a molecule-fixed axis (typically, the largest polarizability axis) along a laboratory-fixed axis and orientation refers to the molecular dipole moments pointing in a particular direction. Alignment and orientation occur in the adiabatic limit where the laser field, used to align the molecules, is turned on and off slowly compared to the inherent rotational periods of the molecule [41, 42]. The state selection leads to strong enhancement in the degree of orientation and alignment of iodobenzene molecules compared to that achieved when no deflection is used.

\section{EXPERIMENTAL}

A schematic of the experimental setup is shown in Figure 1. The molecular beam machine consists of three differentially pumped vacuum chambers; The source cham-

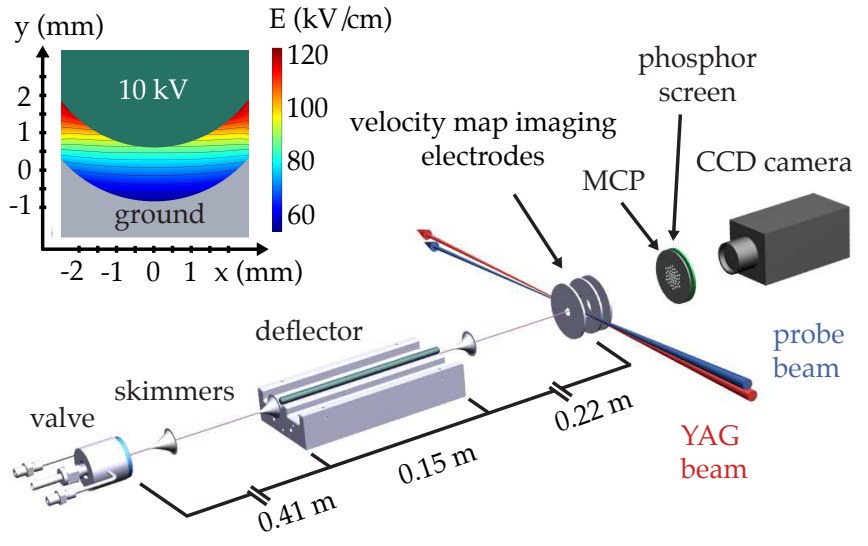

FIG. 1: (Color online): Scheme of the experimental setup. In the inset, a cut through the deflector is shown, and a contourplot of the electric field strength is given. Details of the velocity map imaging spectrometer are shown in Figure 7. See text for details.

ber housing a pulsed valve (pumped by a $2000 \mathrm{l} / \mathrm{s}$ turbomolecular pump), the deflector chamber (pumped by a $500 \mathrm{l} / \mathrm{s}$ turbomolecular pump) and the detection chamber housing the ion/electron spectrometer (pumped by a $500 \mathrm{l} / \mathrm{s}$ turbomolecular pump). About 3 mbar of iodobenzene (Sigma Aldrich, $98 \%$ purity) or benzonitrile (Sigma Aldrich, $98 \%$ purity) is seeded in an inert carrier gas and expanded through a pulsed valve into vacuum. In order to obtain optimal cooling of the molecular beam, a miniaturized, high pressure Even-Lavie valve [37] is used operating at a backing pressure of 90 bar of $\mathrm{He}$ or 20 bar of $\mathrm{Ne}$, limited by the onset of cluster formation. While rotational temperatures down to $0.4 \mathrm{~K}$ have been achieved under similar conditions [43], the typical rotational temperature in our experiments is $\sim 1 \mathrm{~K}$. Two 1-mm-diameter skimmers placed $15 \mathrm{~cm}$ (separating the source and the deflector chamber) and $38 \mathrm{~cm}$ downstream from the nozzle collimate the molecular beam before it enters a 15-cm-long electrostatic deflector. A cut through the electrodes of the deflector is shown in the inset of Figure 1 together with the electric field created. A trough with an inner radius of curvature of $3.2 \mathrm{~mm}$ at ground potential and a rod with a radius of $3.0 \mathrm{~mm}$ at high voltage create a two-wire field [44]. The vertical gap across the molecular beam axis is $1.4 \mathrm{~mm}$, while the smallest distance between the electrodes is $0.9 \mathrm{~mm}$. The two-wire field geometry is ideally suited for molecular beam deflection. The gradient of the electric field along the vertical direction is large and nearly constant over a large area explored by the molecular beam, while the electric field is very homogeneous along the horizontal direction. Thus, a polar molecule experiences a nearly constant force in the vertical direction independent of its position within the deflector, while the force in the horizontal direction (i. e., broadening of the beam in the horizontal direction) is minimized. In our setup, the deflector is mounted such that molecules in high-field-seeking 
(low-field-seeking) quantum states are deflected upwards (downwards).

After passing through the deflector, the molecular beam enters the differentially pumped detection chamber via a third skimmer of $1.5 \mathrm{~mm}$ diameter. In the detection area, the molecular beam is crossed by one or two laser beams that are focused by a spherical lens with a focal length of $f=300 \mathrm{~mm}$. The lens is mounted on a vertical translation stage so that the height of the laser foci can be adjusted with high precision. In the first part of the experiment, where the beam deflection of iodobenzene and benzonitrile is characterized, only one laser, the probe laser, is used. This Ti:Sapphire laser (25 fs (FWHM) pulses, $800 \mathrm{~nm}$, beam-waist $\omega_{0}=21 \mu \mathrm{m}$ ) is used to determine the relative density in the molecular beam via photoionization. In the second part of the experiment, an additional laser pulse is included to study laser induced alignment and orientation of iodobenzene. For these experiments, $10 \mathrm{~ns}$ (FWHM) long pulses from a Nd:YAG laser $\left(1064 \mathrm{~nm}, \omega_{0}=36 \mu \mathrm{m}\right)$ are overlapped in time and space with the probe laser pulses. While the YAG laser induces adiabatic alignment and orientation, here the fs-laser is used to determine the spatial orientation of the target molecules via Coulomb explosion. Ionic fragments produced in the Coulomb explosion are accelerated in a velocity focusing geometry towards the detector. The detector can be gated with a time resolution of $\sim 90 \mathrm{~ns}$, which allows for mass selective detection of individual fragments. A microchannel plate (MCP) detector backed by a phosphor screen is employed to detect the position of mass-selected ions. In particular, $\mathrm{I}^{+}$fragment ions, formed in the Coulomb explosion of iodobenzene, are particularly useful experimental observables since they recoil along the C-I symmetry axis of the molecule. Thus, $2 \mathrm{D}$ ion images of $\mathrm{I}^{+}$recorded with a CCD camera provide direct information about the instantaneous molecular orientation of the C-I bond axis with respect to the laboratory frame and are, therefore, the basic observables in these experiments. All experiments are conducted at $20 \mathrm{~Hz}$, limited by the repetition rate of the YAG laser.

\section{RESULTS AND DISCUSSION}

In the first experiments shown in Section III A, a detailed analysis of electrostatic beam deflection is presented. It is shown, that electrostatic deflection of cold molecular beams can be used to determine the rotational temperature of a supersonic jet. Furthermore, the degree of quantum state selectivity that can be achieved with our setup is investigated. As an application it is demonstrated, how this quantum state selectivity of the deflection process can be exploited to obtain an unprecedented degree of laser induced alignment (Section III B) and orientation (Section III C) of iodobenzene molecules.

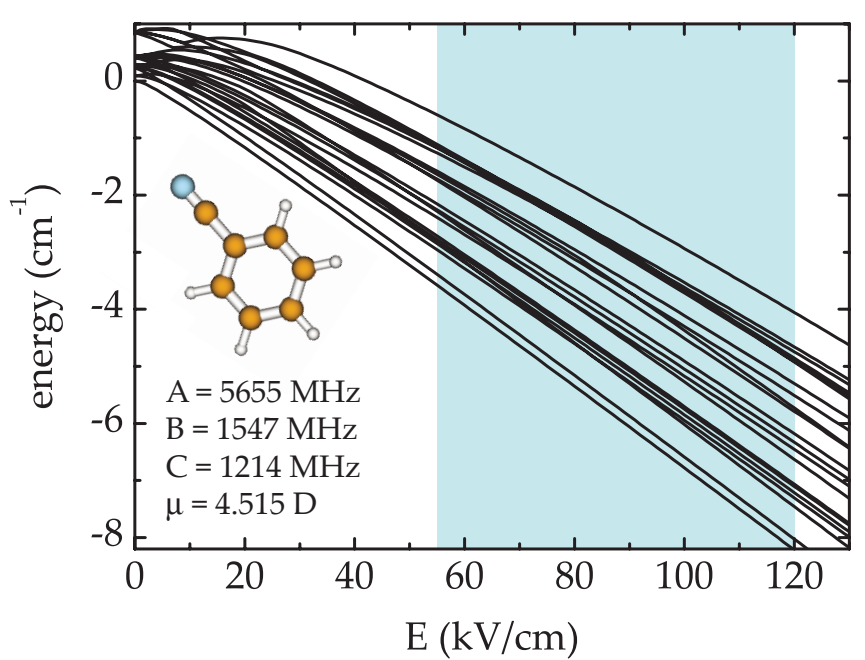

FIG. 2: (Color online): Energy as a function of the electric field strength for the lowest rotational quantum states of benzonitrile. The shaded area indicates the actual electric field strength inside the deflector. In the inset, the molecular structure is shown together with the relevant molecular constants [45].

\section{A. Electrostatic Deflection of Cold Molecular Beams}

In the first experiment, the deflection of benzonitrile molecules $\left(\mathrm{BN}, \mathrm{C}_{7} \mathrm{H}_{5} \mathrm{~N}\right)$ seeded in 90 bar of $\mathrm{He}$ is investigated. $\mathrm{BN}$ is an ideal candidate for electrostatic beam deflection due to its large permanent dipole moment of 4.515 D. From the precisely known molecular constants [45] the energy of a given rotational quantum state can be calculated as a function of the electric field strength. The exact procedure is detailed in Section A 1. Figure 2 shows the Stark energies for the lowest rotational states of BN. Due to the small rotational constants and the resulting high density of rotational states, a large number of states is populated even under the cold conditions in a supersonic expansion. At a rotational temperature of $1 \mathrm{~K}$, the typical temperature in our experiments (vide infra), 66 rotational quantum states (with $419 \mathrm{M}$ components) have a population larger than $1 \%$ relative to the ground state. At the electric field strengths present in the deflector, indicated by the shaded area in Figure 2, all low-lying quantum states are high-field seeking. This is due to mixing of closely spaced states of the same symmetry and is typical for large asymmetric top molecules. The Stark shift and thus the force a molecule experiences in an inhomogeneous electric field depends on the rotational quantum state. Molecules in the ground state have the largest Stark shift and are, therefore, deflected the most. In general, the Stark shift decreases with increasing $J$ quantum number. Thus, the lower the rotational temperature of the molecular beam is, the more the beam is deflected. 


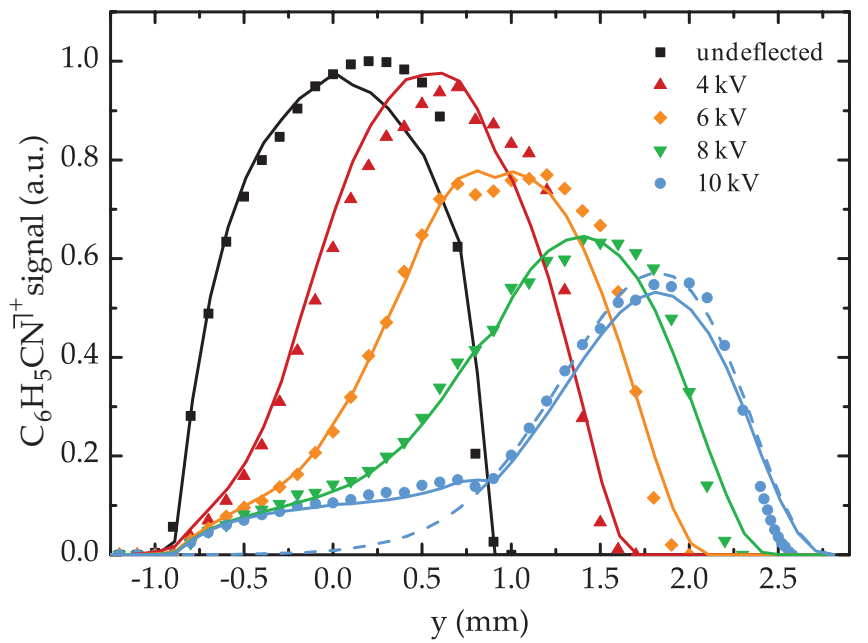

FIG. 3: (Color online): The vertical spatial profile of the molecular beam for different deflection voltages applied, measured by recording the laser induced $\mathrm{BN}^{+}$signal (see text). The experimental data are shown as symbols together with the corresponding simulated profiles (lines).

Figure 3 shows vertical intensity profiles of BN for various high voltages applied to the deflector. Vertical intensity profiles are obtained by recording the $\mathrm{BN}^{+}$signal from photoionization by the fs-laser as a function of the vertical position of the laser focus. If no high-voltage is applied to the deflector, the molecular beam extends over about $\sim 2 \mathrm{~mm}$. In this case, the size of the molecular beam in the detection region is determined by the mechanical aperture of the experimental setup, i. e., by the dimensions of the deflector and the last skimmer before the detection region. As the high voltage is turned on, the molecular beam profile broadens and shifts upwards. At a voltage of $10 \mathrm{kV}$, a large fraction of the molecules is deflected out of the original, undeflected beam profile. A small fraction of the molecules, however, is almost unaffected by the deflector.

In order to understand these experimental findings, Monte Carlo simulations are employed, which are described in detail in Section A 2. In brief, trajectory calculations are performed for molecular packets of individual rotational quantum states. These calculations yield single-quantum-state deflection profiles. Then, the single-state profiles are averaged according to the populations of the respective states in the original molecular beam (i.e., at the entrance of the deflector). From these simulations it is obvious, that the molecules in the original beam are not rotationally thermalized, an effect that has previously been observed in rotationally resolved spectroscopy $[46,47]$. A number of different descriptions of the populations of rotational states have been given [47]; we use the formula for a twotemperature model originally proposed by Levy and coworkers [46]. For details and the approximation of the

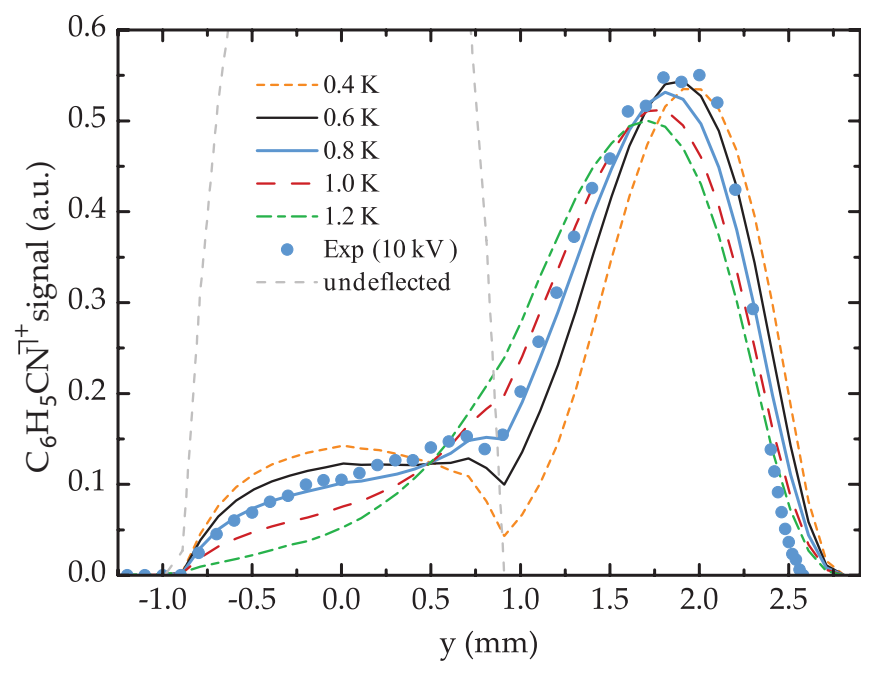

FIG. 4: (Color online): The vertical profile of the molecular beam measured by recording the laser induced $\mathrm{BN}^{+}$signal (see text). The experimental data are shown together with the corresponding simulated profiles (solid lines) for different rotational temperatures. Simulations are shown for the twotemperature model; see text for details.

high-temperature component see Section A 3. Finally, the rotational temperature of the low-temperature component in the molecular beam is obtained by fitting the simulated deflection profiles to the experimental data using a local optimization algorithm. All deflection profiles measured at the different voltages are fitted simultaneously, where the fraction $q$ of the low-temperature component, a general intensity scaling factor $s$ of the deflected profiles (with respect to the undeflected beam profile), and the rotational temperature $T_{\text {rot }}$ of the lowtemperature component are the fitting parameters. Best agreement between experimental data and simulations is found for $q=0.93$ and $T_{\text {rot }}=0.8 \mathrm{~K}$. The resulting simulated deflection profiles nicely reproduce the experimental data as shown in Figure 3 (solid lines). In particular, the undeflected part of the molecular beam for $10 \mathrm{kV}$ can be perfectly simulated, which indicates that the use of a two-temperature model was indeed justified. For comparison, also a simulated deflection profile for $10 \mathrm{kV}$ using a one-temperature model is shown (dashed line in Figure 3).

In order to estimate the uncertainty of $T_{\text {rot }}$, deflection profiles are calculated for different rotational temperatures. For each fixed rotational temperature, the best values for $s$ and $q$ are determined using the fitting procedure outlined above and the resulting deflection profile for a voltage of $10 \mathrm{kV}$ is plotted in Figure 4 . With increasing $T_{\text {rot }}$, the peak of the beam profile shifts towards smaller y-values, while, at the same time, the intensity in the undeflected part of the beam profile is reduced. From the comparison of experimental data and simulation, an uncertainty of $T_{\text {rot }}$ of $\pm 0.2 \mathrm{~K}$ is estimated. 


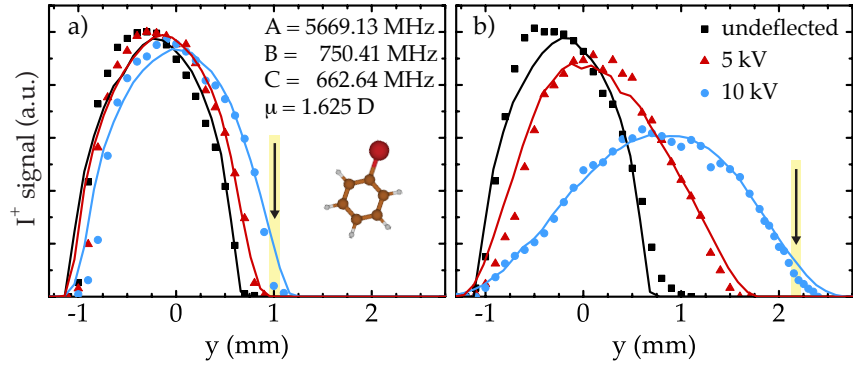

FIG. 5: (Color online): The vertical profile of the molecular beam for different deflection voltages applied, measured by recording the laser induced $\mathrm{I}^{+}$signal (see text). The experimental data are shown together with the corresponding simulated profiles. Figure (a) shows the beam profiles for iodobenzene seeded in 90 bar He, and Figure (b) shows the respective profiles for iodobenzene seeded in 20 bar Ne. Arrows indicate the laser positions for alignment and orientation experiments (vide infra).

The deflection of iodobenzene molecules (IB, $\mathrm{C}_{6} \mathrm{H}_{5} \mathrm{I}$ ) is investigated in the same way. Vertical intensity profiles are measured by recording the signal of $\mathrm{I}^{+}$ions, created by Coulomb explosion with a circularly polarized probe pulse, as a function of the vertical position of the probe laser focus. Figure 5 a) shows deflection measurements for IB seeded in 90 bar of He. IB (mass $204 \mathrm{u}$ ) is heavier than BN (mass $103 \mathrm{u}$ ) and has a considerably smaller dipole moment of only $1.625 \mathrm{D}$ [48] compared to BN. Therefore, smaller deflection amplitudes are observed for IB under identical expansion conditions. However, the interaction time with the electric field and the time-offlight from deflector to detection region can be increased when Ne is used as a carrier gas instead of He. Changing the carrier gas reduces the mean velocity of the molecular beam from $\sim 1800 \mathrm{~m} / \mathrm{s}$ to $\sim 800 \mathrm{~m} / \mathrm{s}$ and significantly enhances the observed deflection as shown in Figure $5 \mathrm{~b}$ ). Following the fitting procedure outlined above, $T_{\text {rot }}$ can be determined for IB as well. In the case of IB, a one-temperature model with a rotational temperature of $1.05 \mathrm{~K}$ fits the experimental data best for the deflection measurements in helium as well as in neon. The uncertainty of $T_{\text {rot }}$ is estimated to be $\pm 0.1 \mathrm{~K}$ for IB seeded in $\mathrm{Ne}$ and $\pm 0.2 \mathrm{~K}$ for IB in He. The somewhat larger uncertainty for the measurements in He reflects the small deflections observed for He. The simulated deflection profiles for IB are shown as solid lines in Figure 5 and agree well with the experimental data.

The main purpose of the deflection studies presented in this work is to provide quantum-state-selected samples of large molecules for further experiments. The degree of deflection that a molecule experiences in the electric field of the deflector depends on its quantum state. The relevant quantity is the effective dipole moment $\mu_{\text {eff }}$ (the negative slope of the Stark curve), which depends on the electric field strength. Molecules residing in low rota- tional quantum states have generally the largest $\mu_{\mathrm{eff}}$ and are, therefore, deflected most. These molecules can simply be addressed by moving the laser focus in the detection region towards the upper cut-off of the molecular beam profile. In order to understand the laser-induced alignment and orientation experiments presented in Section III B and III C, it is crucial to know the relative populations of individual quantum states that are probed at a given height of the laser focus. The positions of the laser foci within the molecular beam profile during the alignment and orientation measurements are indicated by arrows in Figure 5. At this position, the intensity of the deflected beam is $9 \%$ of the undeflected peak intensity. The composition of the molecular packets in the detection region can be extracted from the simulated deflection profiles. Table I provides an overview of the most abundant quantum states present in different regions of the beam profiles for IB at a rotational temperature of $1.05 \mathrm{~K}$. For comparison, also the population of each rotational quantum state in the undeflected beam for this rotational temperature is given. At the position of the laser focus for the orientation experiments in Ne (column four in Table I), the population of the lowest quantum states is significantly enhanced in the deflected beam compared to the undeflected beam. The fraction of ground state molecules is enhanced by a factor of five, for instance. About $97 \%$ of the population resides in the quantum states listed in Table I with the $J_{K_{a} K_{c}}=3_{03}$ state being most abundant. Moving the laser focus even closer towards the upper cut-off in the beam profile should reduce the number of quantum states that are probed even further. If a reduction of the beam intensity by two orders of magnitude (compared to the undeflected beam) can be afforded, only 4 quantum states are predicted to be probed with $37 \%$ of the molecules being in the $J_{K_{a} K_{c}}=3_{03}$ state. At first glance, it is surprising that this state and not the absolute ground state, which is expected to have the largest $\mu_{\mathrm{eff}}$, is populated most in the deflected beam. In order to understand this, the Stark curves for the most deflected quantum states of IB are shown in Figure 6 a), together with their effective dipole moments (Figure $6 \mathrm{~b}$ ). Below the relevant electric field strengths, both the $J_{K_{a} K_{c}} M=3_{03} 2$ and the $J_{K_{a} K_{c}} M=4_{13} 3 \mathrm{M}$-sublevels have avoided crossings with close-by states of the same symmetry (dashed lines in Figure 6 a). These avoided crossings lead to large local effective dipole moments that are comparable to the ground-state $\mu_{\text {eff. }}$ [79] Thus molecules in these quantum states are deflected as much as ground-state molecules. Furthermore, states with $M \neq 0$ are doubly degenerate, whereas the ground state with $M=0$ is only singly degenerate. Therefore, the population of molecules in the $J_{K_{a} K_{c}} M=3_{03} 2$ in the undeflected beam is already larger than the population in the ground state. [80] From Table I it is clear, that it will be difficult to isolate the rotational ground state of iodobenzene in our setup. Never- 


\begin{tabular}{|c|c|c|c|c|c|c|c|c|}
\hline \multicolumn{2}{|c|}{$J_{K_{a} K_{c}} M$} & \multicolumn{2}{|c|}{ IB in He at $0.01 \cdot I_{\text {peak }}$} & \multicolumn{2}{|c|}{ IB in Ne at $0.01 \cdot I_{\text {peak }}$} & $\begin{array}{l}\text { IB in } \mathrm{Ne} \\
P_{M}(\%) \\
\end{array}$ & $\begin{array}{l}\quad 0.09 \cdot I_{\text {peak }} \\
\Sigma P_{M}(\%) \\
\end{array}$ & \begin{tabular}{|c} 
undeflected beam \\
$P_{J_{K_{a} K_{c}}}^{\text {free }}(\%)$ \\
\end{tabular} \\
\hline $0_{00}$ & 0 & 17.51 & 17.51 & 26.06 & 26.06 & 6.24 & 6.24 & 1.15 \\
\hline \multirow{2}{*}{$1_{01}$} & 0 & 0.02 & \multirow{2}{*}{7.71} & & & 3.90 & \multirow{2}{*}{13.61} & \multirow{2}{*}{3.24} \\
\hline & 1 & 7.69 & & & & 9.70 & & \\
\hline \multirow{2}{*}{$1_{11}$} & 0 & 1.92 & \multirow{2}{*}{17.58} & \multirow[b]{2}{*}{23.84} & \multirow{2}{*}{23.84} & 2.37 & \multirow{2}{*}{7.79} & \multirow{2}{*}{1.55} \\
\hline & 1 & 15.66 & & & & 5.43 & & \\
\hline \multirow{2}{*}{$1_{10}$} & 0 & 1.81 & \multirow{2}{*}{1.82} & & & 2.41 & \multirow{2}{*}{6.26} & \multirow{2}{*}{1.55} \\
\hline & 1 & 0.02 & & & & 3.86 & & \\
\hline \multirow{2}{*}{$2_{02}$} & 1 & & & & & 0.57 & \multirow{2}{*}{7.74} & \multirow{2}{*}{4.75} \\
\hline & 2 & & & & & 7.17 & & \\
\hline \multirow{3}{*}{$2_{12}$} & 0 & & \multirow{3}{*}{3.22} & & & 0.23 & \multirow{3}{*}{7.90} & \multirow{3}{*}{2.28} \\
\hline & 1 & & & & & 3.51 & & \\
\hline & 2 & 3.22 & & & & 4.16 & & \\
\hline \multirow{4}{*}{$3_{03}$} & 0 & 0.01 & \multirow{4}{*}{29.73} & & & 2.93 & & \\
\hline & 1 & 6.17 & & & 36.61 & 7.17 & 19.00 & 5.47 \\
\hline & 2 & 23.55 & & 36.61 & & 8.01 & & \\
\hline & 3 & & & & & 0.89 & & \\
\hline $3_{13}$ & 2 & & & & & 0.20 & 298 & 265 \\
\hline 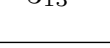 & 3 & & & & & 2.79 & 2.50 & 2.00 \\
\hline 4 & 0 & 0.01 & 309 & & & 2.12 & 768 & 5.43 \\
\hline .04 & 3 & 3.98 & 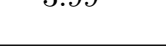 & & & 5.56 & 1.00 & 0.40 \\
\hline & 1 & & & & & 2.12 & & \\
\hline $4_{13}$ & 2 & 2.17 & 10.52 & & 13.49 & 2.60 & 7.58 & 2.54 \\
\hline & 3 & 8.35 & & 13.49 & & 2.87 & & \\
\hline & 1 & & & & & 0.19 & & \\
\hline $5_{05}$ & 2 & & & & & 3.24 & 6.73 & 4.80 \\
\hline & 4 & & & & & 3.30 & & \\
\hline $5_{14}$ & 0 & & 1.66 & & & 0.08 & 1.90 & 2.22 \\
\hline & 4 & 1.66 & & & & 1.83 & & \\
\hline $5_{23}$ & 3 & 0.06 & 5.67 & & & & & 1.92 \\
\hline & 4 & 5.61 & & & & & & 1.22 \\
\hline $6_{16}$ & 2 & & & & & 0.19 & 1.26 & 1.91 \\
\hline & 3 & & & & & 1.07 & & \\
\hline$\Sigma$ & 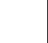 & & 99.41 & & 100.00 & & 96.67 & 41.46 \\
\hline
\end{tabular}

TABLE I: Relative population of individual quantum states in the deflected part of the molecular beam profile for $T_{\text {rot }}=1.05 \mathrm{~K}$. Left: IB in He at $1 \%$ of peak intensity of undeflected beam. Center: IB in Ne at $1 \%$ of peak intensity of undeflected beam. Right: IB in Ne at $9 \%$ of peak intensity of undeflected beam (here orientation images were taken). $P_{M}$ denotes relative population of individual $M$-sublevels in \%, $P_{J_{K_{a} K_{c}}}$ the sum over all $M$-sublevels, and $P_{J_{K_{a} K_{c}}}^{\text {free }}$ the relative population of a given rotational quantum state in a free jet.

theless, given that the fraction of ground-state molecules could be increased from $1 \%$ in the undeflected to $26 \%$ in the deflected beam, dramatic effects are to be expected for a variety of further experiments.

We point out, that we are assuming adiabatic following of potential energy curves in all simulations. Nonadiabatic transitions are unlikely in the strong fields inside the deflector, since the number of avoided crossings and their energy gaps generally increase with electric field strength. Moreover, the probability for non-adiabatic following depends on the rate of change of the field strength, which is only due to the slow translational motion of the molecules. However, non-adiabatic transitions have been observed in different Stark decelerator beamlines at real [49] and avoided crossings [50] for small electric fields. Similarly, when the deflected molecules in the experiments reported here enter a field-free region, scrambling of population over the various $M$ components of their rotational state will occur. 

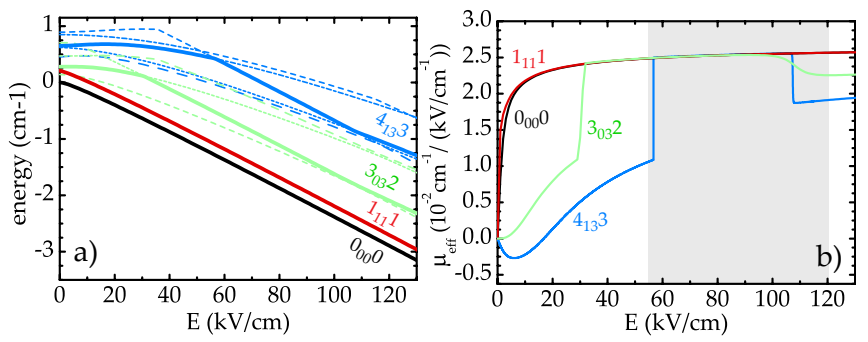

FIG. 6: (Color online): (a) Energy as a function of the electric field strength for selected quantum states of IB. Solid lines represent quantum states that are present close to the upper cut-off of the molecular beam profile for IB seeded in Ne. The respective $J_{K_{a} K_{c}}$ quantum numbers are given in the figure. (b) Effective dipole moment for selected quantum states of IB. The shaded area represents the range of electric field strengths in the deflector at $10 \mathrm{kV}$.

For small molecules, like OCS or $\mathrm{ClCN}$, the preparation of an ensemble of molecules, all in a single quantum state will be feasible with the present setup. For these systems, the number of quantum states that are populated in a supersonic jet is significantly smaller compared to large asymmetric top molecules like IB or BN. The spacing between neighboring quantum states is larger and the number of avoided crossings smaller. Thus, the differences in the effective dipole moment between individual quantum states are larger and, therefore, the degrees of their deflection will vary considerably.

\section{B. Laser Induced Alignment of Quantum-State-Selected Molecules}

We now turn to studying alignment induced by the YAG pulse. The basic experimental observables are 2D $\mathrm{I}^{+}$ion images recorded when the iodobenzene molecules are irradiated with both the YAG pulse and the probe pulse. The geometry of the laser pulse polarizations with respect to the velocity map imaging spectrometer (VMI) is illustrated in Figure 7. The YAG pulse is linearly polarized along the vertical direction, i. e., in the detector plane. The probe pulse is linearly polarized perpendicular to the detector plane, which ensures that there is no detection bias on the molecular orientation in that plane. This results in a circularly symmetric $\mathrm{I}^{+}$image, when only the probe pulse is used (Figure $8 \mathrm{~A} 1$ ). When the YAG pulse is included (Figure $8 \mathrm{~A} 2-\mathrm{A} 4$ ) the $\mathrm{I}^{+}$images exhibit strong angular confinement along the polarization of the YAG pulse.

The $\mathrm{I}^{+}$ions appear as two pairs of radially localized regions, corresponding to two different fragmentation channels of the Coulomb explosion. The radius of the outermost (and weakest) pair of rings is approximately $\sqrt{2}$

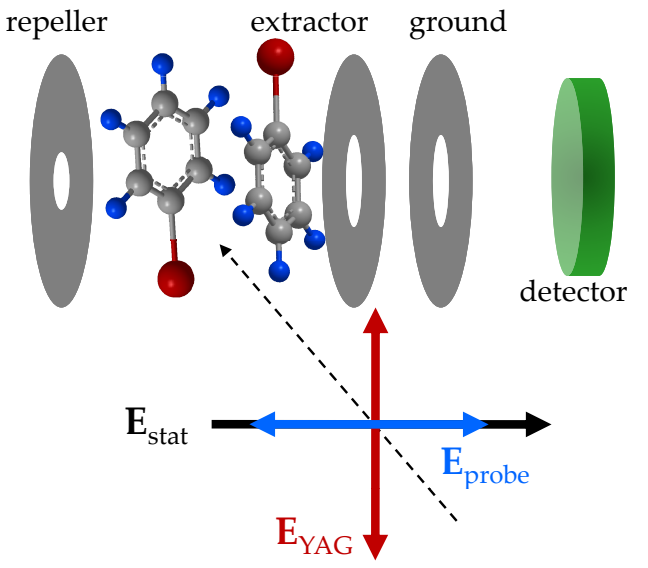

FIG. 7: (Color online) Schematic illustration of the polarization state of the YAG and the probe pulse with respect to the static electric field and the detector plane used to characterize alignment. The dashed line represents the propagation directions of the laser beams. Included is also a sketch of the resulting molecular alignment. Repeller, extractor and ground refers to the electrostatic plates of the velocity map imaging spectrometer.

times larger than the radius of the innermost (and brightest) pair of rings. Since the radius is proportional to the velocity of the ions the $\mathrm{I}^{+}$ions from the outermost pair of rings originate from a Coulomb explosion channel that releases twice as much kinetic energy as the channel producing the $\mathrm{I}^{+}$ions in the innermost pair of rings. As pointed out in several previous studies from our group (see for instance reference 51) this is only consistent with the innermost pair of rings originating from iodobenzene being doubly ionized by the probe pulse and fragmenting

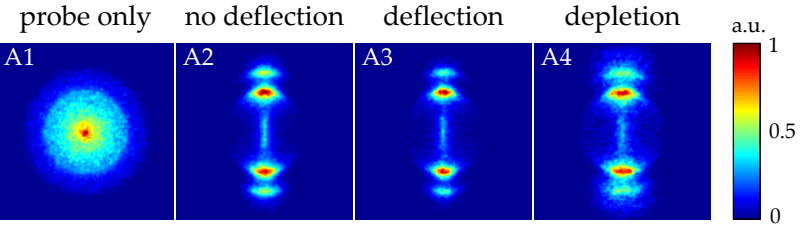

FIG. 8: (Color online) $\mathrm{I}^{+}$ion images illustrating alignment, recorded when the probe pulse Coulomb explodes the iodobenzene molecules seeded in He. The polarizations of the YAG and the probe pulses are kept fixed as illustrated in Figure 7. The labels "no deflection", "deflection" and "depletion" correspond to images recorded at lens position $y=0.0 \mathrm{~mm}, 1.0 \mathrm{~mm}$ and $-0.9 \mathrm{~mm}$ respectively, the latter two with the deflector at $10 \mathrm{kV}$ (see Figure 5). The intensities of the YAG and probe pulse are $8 \times 10^{11} \mathrm{~W} / \mathrm{cm}^{2}$ and $5 \times 10^{14} \mathrm{~W} / \mathrm{cm}^{2}$, respectively. The color scale indicates the relative number of ions. This color scale is the same for all subsequent figures showing ion images. 


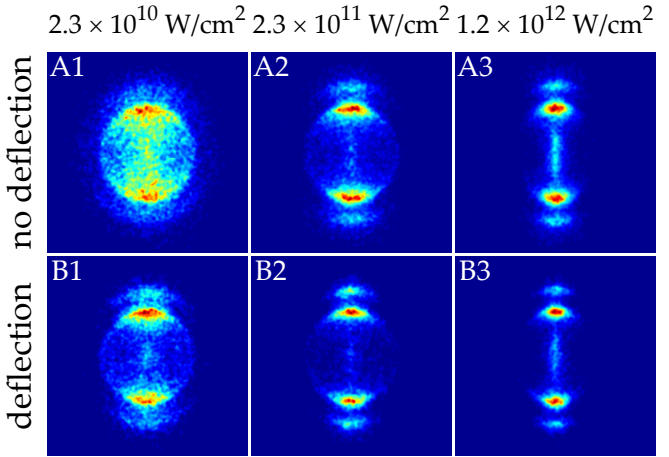

FIG. 9: (Color online) $\mathrm{I}^{+}$ion images illustrating alignment at different intensities of the YAG pulse, recorded when the probe pulse Coulomb explodes iodobenzene molecules seeded in 20 bar Ne. The labels "no deflection" and "deflection" correspond to images recorded at lens position $y=0.0 \mathrm{~mm}$ (deflector turned off) and $2.15 \mathrm{~mm}$ (deflector at $10 \mathrm{kV}$ ), respectively. The intensity of the probe pulse is $5 \times 10^{14} \mathrm{~W} / \mathrm{cm}^{2}$.

into an $\mathrm{I}^{+}+\mathrm{C}_{6} \mathrm{H}_{5}{ }^{+}$ion pair, and the outermost pair of rings originating from $\mathrm{I}^{+}$ions formed by triple ionization and fragmentation into an $\mathrm{I}^{+}+\mathrm{C}_{6} \mathrm{H}_{5}{ }^{2+}$ ion pair. The pronounced angular confinement observed in images A2A4 is quantified by calculating the expectation value of $\left\langle\cos ^{2} \theta_{2 D}\right\rangle$, where $\theta_{2 D}$ is the angle between the YAG pulse polarization and the projection of the $\mathrm{I}^{+}$recoil velocity vector onto the detector plane. In this paper, $\left\langle\cos ^{2} \theta_{2 D}\right\rangle$ values are calculated only from ions detected in radial region corresponding to the $\mathrm{I}^{+}+\mathrm{C}_{6} \mathrm{H}_{5}{ }^{2+}$ channel. By doing so, the YAG intensities probed are restricted to a narrow range close to the maximum value, as the high nonlinearity of the multiphoton process occurs efficiently only in the spatial regions close to the focal point of the YAG beam.

Image A1, recorded with only the probe pulse present, should correspond to a target of randomly oriented molecules. As expected the image is circular symmetric and $\left\langle\cos ^{2} \theta_{2 D}\right\rangle=0.515$. When the YAG pulse is included (image A2) a pronounced angular confinement is observed along the polarization of the YAG pulse and $\left\langle\cos ^{2} \theta_{2 D}\right\rangle$ is increased to 0.947 . These observations are in complete agreement with previous studies [38]. When the deflector is turned on and the laser foci moved to the edge of the most deflected molecules (at the position marked in Figure $5 \mathrm{a}$ ), corresponding to molecules in the lowest rotational states, the angular confinement is further enhanced (image A3) leading to a $\left\langle\cos ^{2} \theta_{2 D}\right\rangle$ value of 0.968 . By contrast, when the experiment is conducted on the least deflected molecules in the depleted region (image A4), corresponding to molecules in the highest rotational states, the alignment is weakened and $\left\langle\cos ^{2} \theta_{2 D}\right\rangle=0.900$.

We repeated the alignment measurements when iodobenzene was seeded in Ne. The results are displayed in Figure 9. Like in the He case a pronounced improve-

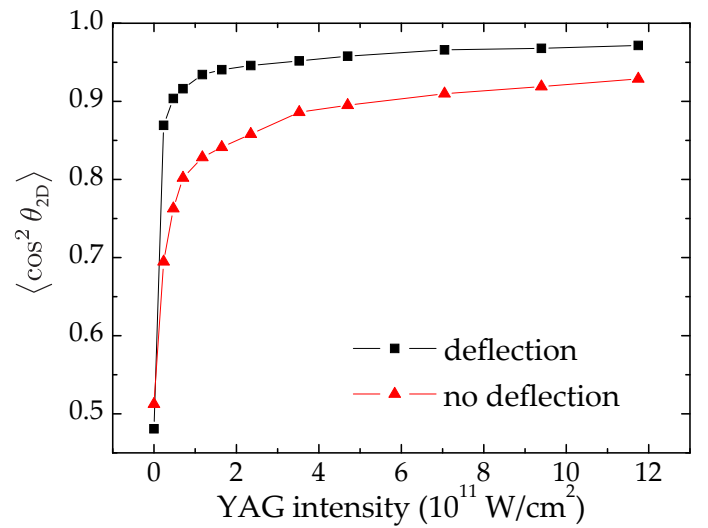

FIG. 10: Degree of alignment as a function of the YAG intensity for iodobenzene seeded in 20 bar Ne. The labels "no deflection" and "deflection" correspond to images recorded at lens position $y=0.0 \mathrm{~mm}$ (deflector turned off) and $2.15 \mathrm{~mm}$ (deflector at $10 \mathrm{kV}$ ), respectively. The intensity of the probe pulse is $5 \times 10^{14} \mathrm{~W} / \mathrm{cm}^{2}$.

ment is observed when deflected rater than undeflected molecules are employed. Figure 9 shows images of $\mathrm{I}^{+}$ recorded at three different intensities of the YAG laser for both undeflected and deflected molecules seeded in Ne. The effect of the deflector is clearly seen when comparing, for instance, image B1 (deflected) and A1 (undeflected). At this low YAG intensity $\left(2.3 \times 10^{10} \mathrm{~W} / \mathrm{cm}^{2}\right)$ weak alignment is obtained in the non-deflected beam with $\left\langle\cos ^{2} \theta_{2 D}\right\rangle=0.695$. Going to the edge of the deflected molecular beam (position indicated in Figure 5 b ) a clear enhancement is observed (image B1) with the $\left\langle\cos ^{2} \theta_{2 D}\right\rangle$ value rising to 0.869 . Also, at high YAG intensity $\left(1.2 \times 10^{12} \mathrm{~W} / \mathrm{cm}^{2}\right)$ the difference in angular confinement comparing the undeflected (image A3) to the deflected molecules (image B3) is visible. While $\left\langle\cos ^{2} \theta_{2 D}\right\rangle=0.929$ represents the limit of the degree of alignment of iodobenzene seeded in $\mathrm{Ne}$ in the undeflected beam, emloying the deflector leads to an unprecedented degree of laser-induced alignment of $\left\langle\cos ^{2} \theta_{2 D}\right\rangle=0.972$. We note that $\left\langle\cos ^{2} \theta_{2 D}\right\rangle=1$ would correspond to the quantum mechanically unfeasible situation of perfectly $1 \mathrm{D}$ aligned molecules.

To quantify the angular information of the images, $\left\langle\cos ^{2} \theta_{2 D}\right\rangle$ values are plotted as a function of YAG intensity, the results are displayed in Figure 10. Even at very low laser intensities a high degree of alignment can be obtained from an ensemble of quantum-state-selected molecules. The tendency shown in this graph with a steep rise and early saturation of the degree of alignment agrees with previous results investigating the dependence of alignment on the rotational temperature of the ensemble of molecules [38]. Effectively, the quantum-stateselection corresponds to a "colder" albeit non-thermal beam (see Section III A). 


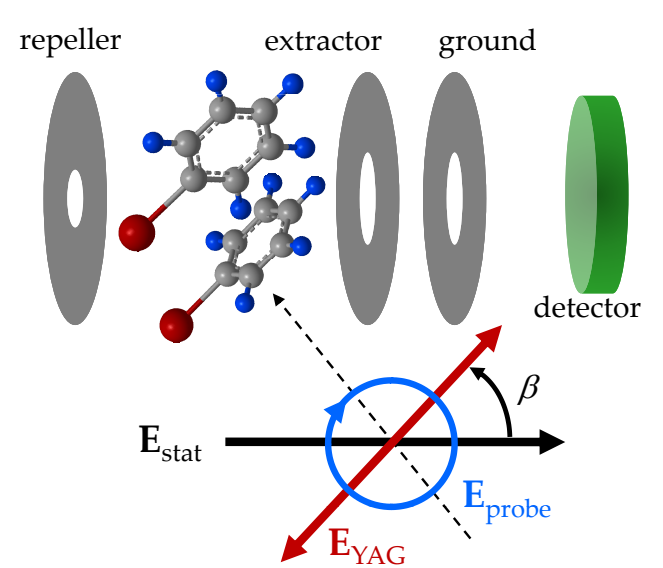

FIG. 11: (Color online) Schematic illustration of the polarization state of the YAG and the probe pulse with respect to the static electric field and the detector plane used to characterize orientation. The dashed line represents the propagation direction of the laser beams. Also, a sketch illustrating the molecular orientation is included.

Note that the contrast between the undeflected and the deflected beam is expected to be greater if $\mathrm{Ne}$ is used instead of $\mathrm{He}$ as a carrier gas. In the undeflected beam the maximum degree of alignment that can be achieved is smaller in Ne because the rotational cooling in the supersonic expansion is less effective due to the lower stagnation pressure[37]. Additionally, in the deflected beam a better degree of alignment is expected for $\mathrm{Ne}$, as the efficiency of the quantum-state-selection in the present setup is significantly enhanced due to the longer residence time in the deflector (see Section III A).

\section{Laser Induced Orientation of Quantum-State-Selected Molecules}

Next, we discuss orientation due to the combined action on the molecules by the YAG pulse and the static electric field $\left(\mathrm{E}_{\text {stat }}\right)$ from the VMI electrodes $[5,6]$. Figure 11 illustrates the polarization state of the YAG and the probe pulse with respect to the static electric field of the VMI electrodes. The important difference compared to the alignment data is that the YAG polarization is rotated away from the axis perpendicular to the static field. Thus, the orientation data result from geometries where the angle $\beta$, between the YAG polarization (the C-I bond axis) and the static electric field, is different from $90^{\circ}$ - see Figure 11. To image orientation a circularly polarized probe pulse is used. This ensures that any molecule will be ionized - and thus detected - with the same probability independent of $\beta$. This circularly polarized probe will induce some bias on the angular dis-

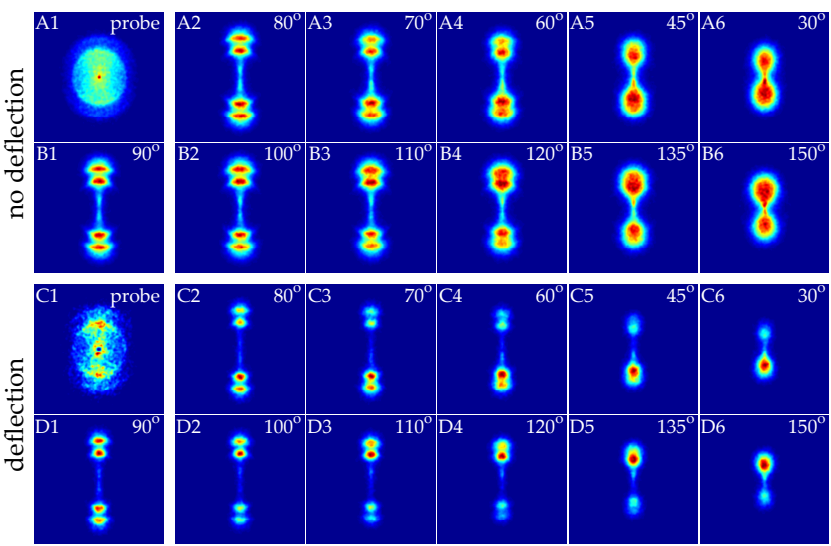

FIG. 12: (Color online) $\mathrm{I}^{+}$ion images illustrating orientation for different values of $\beta$, recorded when the circularly polarized probe pulse Coulomb explodes the iodobenzene molecules seeded in 90 bar He. The labels "no deflection" and "deflection" correspond to images recorded at lens position $y=0.0 \mathrm{~mm}$ (deflector turned off) and $1.0 \mathrm{~mm}$ (deflector at $10 \mathrm{kV}$ ), respectively. The intensity of the YAG and the probe pulse is $8 \times 10^{11} \mathrm{~W} / \mathrm{cm}^{2}$ and $5 \times 10^{14} \mathrm{~W} / \mathrm{cm}^{2}$, respectively. $\mathrm{E}_{\text {stat }}=594 \mathrm{~V} / \mathrm{cm}$.

tribution of the $\mathrm{I}^{+}$ions (see Figure $12 \mathrm{~A} 1$ and $\mathrm{C} 1$ ), however, importantly, it is up/down symmetric. Figure 12 shows $\mathrm{I}^{+}$ion images for different $\beta$ values for both deflected and undeflected molecules seeded in He. As mentioned the circularly polarized probe alone gives rise to an image that exhibits some angular confinement with $\left\langle\cos ^{2} \theta_{2 D}\right\rangle=0.70$ (Figure $12 \mathrm{~A} 1$ ). Consequently, including the YAG pulse at $\beta=90^{\circ}$ results in an image (Figure 12 B1) that appears slightly different from the corresponding image with a linearly polarized probe (Figure 8 A2), but still shows that the molecules are tightly aligned.

Focussing first on the non-deflected data of Figure 12 (row $\mathrm{A}$ and $\mathrm{B}$ ) two prominent changes are observed as the polarization of the YAG pulse is gradually rotated away from the detector plane (images A2-A6 and B2$\mathrm{B} 6)$. First, the location of the $\mathrm{I}^{+}$rings shifts closer to the center of the images. This is due to the fact that the C-I axis alignment, and thus the emission direction of the $\mathrm{I}^{+}$ions, follows the YAG pulse polarization. When the $\mathrm{C}$-I axis is aligned at an angle $\beta$ the magnitude of the $\mathrm{I}^{+}$velocity vector recorded on the detector will be reduced by the factor $\sin (\beta)$. The detrimental effect on the radial (velocity) resolution is obvious at $\beta=135^{\circ} / 45^{\circ}$ (image A5 and B5) and $30^{\circ} / 150^{\circ}$ (image A6 and B6), where the two $\mathrm{I}^{+}$explosion channels, $\mathrm{I}^{+}+\mathrm{C}_{6} \mathrm{H}_{5}{ }^{+}$and $\mathrm{I}^{+}+\mathrm{C}_{6} \mathrm{H}_{5}{ }^{2+}$, become indistinguishable as they merge in the $2 \mathrm{D}$ projection onto the detector plane.

Secondly, as the YAG pulse polarization is turned away from $90^{\circ}$ the up/down symmetry of the images, characteristic for the alignment data described in Section III B (and Figure 12 column 1), is broken. For images with $90^{\circ}<\beta<180^{\circ}$ (images B2-B6) more $\mathrm{I}^{+}$ions are de- 
tected in the upper part, whereas for $0^{\circ}<\beta<90^{\circ}$ (images A2-A6) more $\mathrm{I}^{+}$ions are detected in the lower part. The asymmetry becomes more pronounced as the YAG polarization is rotated closer to the axis of the static field. We interpret these observations as orientation due to the combined effect of the YAG laser field and the projection of the static electric extraction field $\left(\mathrm{E}_{\mathrm{stat}}\right)$ on the YAG polarization axis. This projection (numerical value: $|\cos (\beta)| \cdot \mathrm{E}_{\text {stat }}$ increases as $\beta$ is rotated towards $0^{\circ}$ or $180^{\circ}$, which is expected to cause an increase of the orientation $[5,6]$, in agreement with the experimental findings.

As discussed in Section III A all states of iodobenzene are high-field seeking, hence the orientation is expected to place the I-end of the molecules towards the repeller plate (see Figure 11), where the electrical potential is highest, because the dipole moment of iodobenzene is directed along the C-I axis pointing from iodine ("negative end") towards the phenyl ring ("positive end"). The expected resulting molecular orientation at a given angle of $\beta$ is shown in Figure 11. Thus, for $0^{\circ}<\beta<90^{\circ}$ the $\mathrm{I}^{+}$ ions are expected to preferentially be ejected downwards, and for $90^{\circ}<\beta<180^{\circ}$ they will be ejected upwards. This is in agreement with the up/down asymmetry in the images.

The alignment (images D1) and orientation (images C2-C6 and D2-D6) improves significantly when the deflector is turned on and the foci of the lasers are moved to the position of the most deflected molecules [position marked in Figure 5 a)]. The markedly better orientation resulting in a much more pronounced up/down asymmetry is clearly visible even when the the YAG pulse is only turned slightly away from perpendicular, i. e., comparing deflected and undeflected images for $\beta=100^{\circ}$ (image C2 and A2) and $\beta=80^{\circ}$ (image D2 and B2). From the previous discussion it appears that the highest degree of orientation is achieved when $\beta$ is rotated towards $0^{\circ}$ or $180^{\circ}$. This is clearly seen from the images in row $\mathrm{C}$ and $\mathrm{D}$ and, again, the improvement obtained with deflected molecules is striking - compare image C6 to A6 (or D6 to B6).

Similar orientation measurements were conducted for iodobenzene seeded in Ne instead of in He. Figure 13 shows $\mathrm{I}^{+}$images at a series of $\beta$ values for two different intensities of the YAG pulse recorded with the deflector at $10 \mathrm{kV}$ at the position marked in Figure $5 \mathrm{~b}$ ). Compared to the images displayed in Figure 12 row A and $\mathrm{B}$, although recorded at slightly different intensities of the YAG pulse, a significant improvement is observed. Furthermore even at low intensity of the YAG a high degree of orientation for iodobenzene seeded in $\mathrm{Ne}$ is achieved. At the same time some loss in the angular confinement, i. e., in the alignment degree, is visible. We assign the clear improvement in the up/down asymmetry to the more stringent state selection in Ne compared to He as described in Section III A, hence, manifesting itself in a higher degree of orientation.
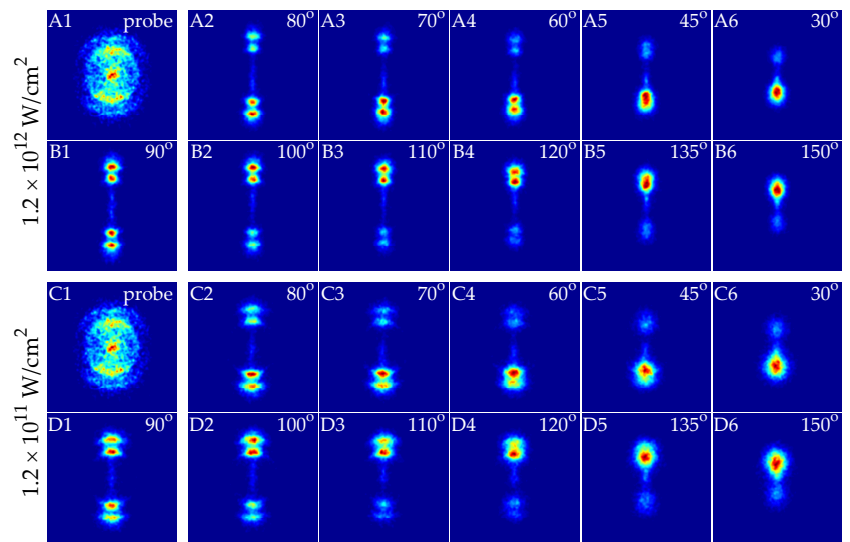

FIG. 13: (Color online) $\mathrm{I}^{+}$ion images of deflected iodobenzene seeded in 20 bar Ne, illustrating orientation at two different intensities of the YAG pulse. The images are recorded at lens position $y=2.25 \mathrm{~mm}$ with the deflector at $10 \mathrm{kV}$. The intensity of the probe pulse is $5 \times 10^{14} \mathrm{~W} / \mathrm{cm}^{2}$. $\mathrm{E}_{\text {stat }}=594 \mathrm{~V} / \mathrm{cm}$.

To quantify the up/down asymmetry, i.e., the degree of orientation, we determine for each image the number of $\mathrm{I}^{+}$ions, $\mathrm{N}\left(\mathrm{I}^{+}\right)_{\text {up }}$, in the upper part of the $\mathrm{I}^{+}+\mathrm{C}_{6} \mathrm{H}_{5}{ }^{+}$and $\mathrm{I}^{+}+\mathrm{C}_{6} \mathrm{H}_{5}{ }^{2+}$ channels (i. e., ions detected in the upper half of the images) as well as the total number of ions, $\mathrm{N}\left(\mathrm{I}^{+}\right)_{\text {total }}\left(=\mathrm{N}\left(\mathrm{I}^{+}\right)_{\text {up }}+\mathrm{N}\left(\mathrm{I}^{+}\right)_{\text {down }}\right)$. This ratio, as a function of $\beta$, is displayed in Figure 14.

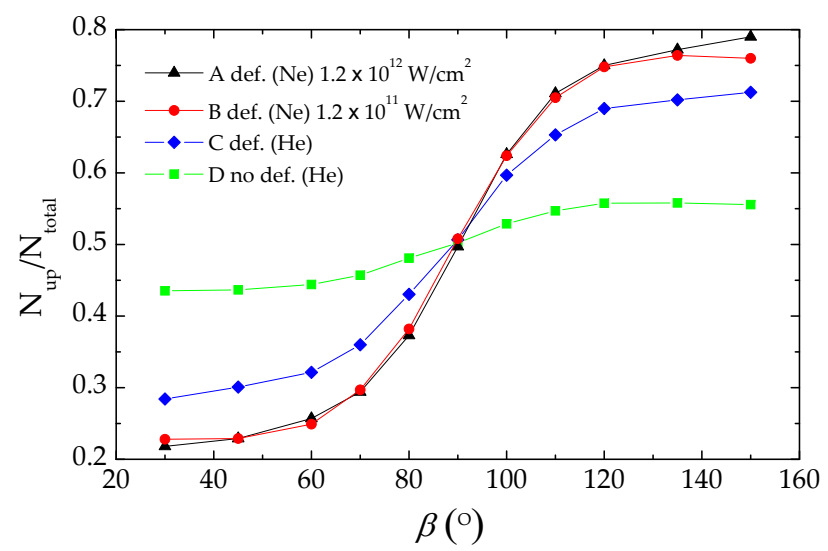

FIG. 14: Orientation of iodobenzene, seeded in either He or $\mathrm{Ne}$, represented by the number of $\mathrm{I}^{+}$ions in the upper half of the image $\left(\mathrm{N}_{\mathrm{up}}\right)$ divided by the total number of $\mathrm{I}^{+}$ions in the image $\left(\mathrm{N}_{\text {total }}\right)$ as a function of $\beta$. For the experiments conducted with $\mathrm{He}$ as a carrier gas, curves $\mathrm{C}$ (lens position $y=1.0 \mathrm{~mm}$ and deflector at $10 \mathrm{kV}$ ) and $\mathrm{D}$ (lens position $y=0.0 \mathrm{~mm}$ and deflector off), the intensity of the YAG pulse was $7.8 \times 10^{11} \mathrm{~W} / \mathrm{cm}^{2}$. For the Ne carrier gas, curves $\mathrm{A}$ and $\mathrm{B}$ (lens position $y=2.25 \mathrm{~mm}$ and deflector at $10 \mathrm{kV}$ ), the intensities of the YAG pulse are displayed in the insert. The intensity of the probe pulse for all curves was fixed at $5 \times 10^{14} \mathrm{~W} / \mathrm{cm}^{2}, \mathrm{E}_{\text {stat }}=594 \mathrm{~V} / \mathrm{cm}$. 
Focussing first on curves $\mathrm{C}$ and $\mathrm{D}$, representing iodobenzene seeded in He, the difference between the data for the deflected molecules and the data obtained with the deflector turned off is striking and shows the advantage of selecting the lowest-lying rotational states for strongly increasing the degree of orientation. The further improvement when $\mathrm{Ne}$ is used instead of He is clear from curves A and $\mathrm{B}$. These two curves also show that the pronounced degree of orientation is maintained when the intensity of the YAG pulse is lowered by an order of magnitude compared to the maximum value of $1.2 \times 10^{12} \mathrm{~W} / \mathrm{cm}^{2}$.

\section{CONCLUSION AND OUTLOOK}

In conclusion, we have shown that deflection of cold molecular beams with an inhomogeneous static electric field enables the selection and the spatial separation of the most polar quantum states, i. e., the lowest-lying rotational states. The method demonstrated here is complementary to state-selection for small molecules using a hexapole focuser, which has been suggested to be applied for improved alignment and orientation experiments [52] and recently been experimentally demonstrated [53]. While a hexapole focuser only works for small molecules in low-field seeking quantum states, beam deflection will apply broadly to a wide range of molecules, from diatomics to large biomolecules. The deflection is strongest for molecules with a large permanent dipole moment to mass ratio. For a given molecule the deflection is optimized by employing stronger deflection fields, increasing the length of the deflector, or lowering the speed of the molecule, for instance, by using neon rather than helium as a carrier gas. For small molecules, the preparation of an ensemble of molecules all in a single quantum state should be feasible. As an application of the state-selected molecules we showed that selection of iodobenzene in low lying rotational states allows to achieve unprecedented degrees of laser-induced adiabatic alignment and mixed laser- and static-field orientation. In particular, we demonstrated that strong alignment and orientation can be maintained even when the intensity of the alignment pulse is lowered to the $10^{10}-10^{11} \mathrm{~W} / \mathrm{cm}^{2}$ range. This can reduce unwanted disturbance from the laser field in future applications of adiabatically aligned or oriented molecules. We note that it should be possible to improve the degree of orientation obtained here simply by increasing the static electric field. Due to experimental constraints this was not implemented in the present work.

Getting access to cold molecules in the gas phase typically involves using a molecular beam from a supersonic expansion that usually consists of more than 99 percent carrier gas and less than one percent of the specific molecules. In several types of experiments the atomic carrier gas can contribute to, or even completely overshadow the particular signal measured. The electrostatic deflection naturally separates the polar molecules from the unpolar carrier gas and thus removes this unwanted background. We conservatively estimate the density in the original molecular beam to be $10^{11}$ molecules $/ \mathrm{cm}^{3}$. In the experiments presented here the density in the deflected part of the beam is approximately $10^{10}$ molecules $/ \mathrm{cm}^{3}$. We foresee this density to be sufficient for a variety of applications, such as photoelectron spectroscopy with VUV, EUV [54], or x-ray light sources, including attosecond pulses, or high harmonic generation experiments with fs laser pulses [55]. For all of these applications the separation of the molecular target from the carrier gas might be of great relevance.

Additionally, brute-force orientation [1, 2], that is, the spatial orientation of polar molecules using strong dc electric fields, will benefit from the state-selected samples similar to what was demonstrated here. In fact, the states that are deflected the most are also oriented the most in a dc electric field. To illustrate the achievable orientation, we have calculated the ensemble averaged orientation in a homogeneous electric field of $250 \mathrm{kV} / \mathrm{cm}$ for iodobenzene for a thermal ensemble of $1 \mathrm{~K}$. For this ensemble $\langle\cos \theta\rangle=0.757$ is obtained. For a deflected, quantum-state-selected sample of iodobenzene molecules at $1 \%$ of the undeflected peak intensity (see Table I), an increased ensemble averaged orientation of $\langle\cos \theta\rangle=0.905$ is predicted. For nonadiabatic alignment $[4,56]$ it was demonstrated, that the dynamics and, importantly, the degree of alignment and orientation depends strongly on the initial rotational state distribution [57-61]. Selection of rotational states is, therefore, highly advantageous for nonadiabatic laser-induced schemes to control the spatial orientation of molecules, as recently demonstrated for NO molecules. [53] In general, complete elimination of the rotational tumbling of an asymmetric top molecule requires, that all three principal axes are confined along laboratory fixed axes. This is the area of 3D alignment and orientation, subjects that have been treated theoretically and experimentally [6267]. A straightforward extension of the current work would be significantly improved 3D alignment and orientation of polar molecules by employing state-selected molecules and we have recently performed such experiments [67].

For large (bio-)molecules, typically multiple structural isomers (conformers) [68] are present even at the low temperatures in a supersonic jet [69]. These conformers often exhibit large, and largely different dipole moments what can be exploited to spatially separate them using inhomogeneous electric fields. This separation has recently been demonstrated in an alternating gradient focusing (AG) selector for the cis- and trans-conformers of 3-aminophenol [7]. However, also the static field of a deflector can be used to spatially isolate individual conformers [70]. In general, the ability to achieve very high 
degrees of alignment and orientation is of great interest for a number of applications. We therefore believe state-selected molecules could be very beneficial for areas such as photoelectron angular distributions from fixed-inspace molecules [71], (ultrafast) diffraction with electron or x-ray sources $[8,9]$ and time-resolved studies of lightinduced stereochemistry [72]. Furthermore, Janssen and coworkers have shown that the ability to select a single rotational state with a hexapole focuser enables new possibilities for studying directional dynamics of fragments in photodissociation of small molecules [26]. The deflection method strongly increases the number of molecules to which single rotational state selection, and subsequent orientation, can be applied. In particular, it will offer access to studies of photoinitiated processes in oriented targets of larger asymmetric tops.

\section{Appendix A: Simulation of Beam Profiles}

In this Appendix, the simulations of the experimental deflection profiles are described in detail. First, the calculation of the Stark effect for asymmetric top molecules is explained in Section A 1. Section A 2 describes how single-quantum state deflection profiles are obtained from trajectory simulations using the calculated Stark curves. Finally, the single-quantum state profiles are averaged in a suitable way to simulate a thermal ensemble for a given rotational temperature, which is described in Section A 3, and fitted to the experimental data (Section A 4).

\section{Stark Effect Calculations}

In order to calculate the adiabatic energy curves for asymmetric top molecules, the Hamiltonian matrix is set up in the basis of symmetric top wavefunctions. In the presence of an electric field, only $M$ is a good quantum number for an asymmetric rotor. $J$, which is a good quantum number in the field-free case, is mixed by the field, whereas $K$ is mixed by the molecular asymmetry. Thus, the adiabatic energy curves can be calculated for the different $M$ levels individually by setting up and diagonalizing the $M$ matrices including all $J$ and $K$ levels. An accurate description of higher rotational quantum states also requires including centrifugal distortion constants. The Hamiltonian $H$ of an asymmetric rotor molecule with dipole moment $\vec{\mu}$ in an electric field of strength $E$ can be written as the sum of the Hamiltonian $H_{\text {rot }}$ of an asymmetric rotor in free space in Watson's A-reduction [73] and the contribution due to the Stark effect $H_{\text {Stark }}$ as

$$
H=H_{\text {rot }}+H_{\text {Stark }}
$$

Following references 73 and 74 , the corresponding matrix elements are: [81]

$$
\begin{aligned}
\left\langle J K M\left|H_{\text {rot }}\right| J K M\right\rangle= & \frac{B+C}{2}\left(J(J+1)-K^{2}\right)+A K^{2} \\
& -\Delta_{J} J^{2}(J+1)^{2}-\Delta_{J K} J(J+1) K^{2}-\Delta_{K} K^{4} \\
\left\langle J K \pm 2 M\left|H_{\text {rot }}\right| J K M\right\rangle= & \left(\frac{B-C}{4}-\delta_{J} J(J+1)-\frac{\delta_{K}}{2}\left((K \pm 2)^{2}+K^{2}\right)\right) \\
& \cdot \sqrt{J(J+1)-K(K \pm 1)} \sqrt{J(J+1)-(K \pm 1)(K \pm 2)} \\
\left\langle J K M\left|\mu_{a}\right| J K M\right\rangle= & -\frac{M K}{J(J+1)} \mu_{a} E \\
\left\langle J+1 K M\left|\mu_{a}\right| J K M\right\rangle= & \left\langle J K M\left|\mu_{a}\right| J+1 K M\right\rangle \\
= & -\frac{\sqrt{(J+1)^{2}-K^{2}} \sqrt{(J+1)^{2}-M^{2}}}{(J+1) \sqrt{(2 J+1)(2 J+3)}} \mu_{a} E \\
\left\langle J K \pm 1 M\left|\mu_{b}\right| J K M\right\rangle= & -\frac{M \sqrt{(J \mp K)(J \pm K+1)}}{2 J(J+1)} \mu_{b} E \\
\left\langle J+1 K \pm 1 M\left|\mu_{b}\right| J K M\right\rangle= & \left\langle J K \pm 1 M\left|\mu_{b}\right| J+1 K M\right\rangle \\
= & \pm \frac{\sqrt{(J \pm K+1)(J \pm K+2)} \sqrt{(J+1)^{2}-M^{2}}}{2(J+1) \sqrt{(2 J+1)(2 J+3)}} \mu_{b} E \\
\left\langle J K \pm 1 M\left|\mu_{c}\right| J K M\right\rangle= & \pm i \frac{M \sqrt{(J \mp K)(J \pm K+1)}}{2 J(J+1)} \mu_{c} E \\
\left\langle J+1 K \pm 1 M\left|\mu_{c}\right| J K M\right\rangle= & \left\langle J K \pm 1 M\left|\mu_{c}\right| J+1 K M\right\rangle \\
= & -i \frac{\sqrt{(J \pm K+1)(J \pm K+2)} \sqrt{(J+1)^{2}-M^{2}}}{2(J+1) \sqrt{(2 J+1)(2 J+3)}} \mu_{c} E
\end{aligned}
$$


For the correct assignment of the states to the "adiabatic quantum number labels" $\tilde{J}_{\tilde{K}_{a} \tilde{K}_{c}} \tilde{M}$, i. e., to the adiabatically corresponding field-free rotor states, one has to classify the states according to their character in the electric field symmetry group $[75,76]$. This symmetry classification can be performed by applying a Wang transformation [77] to the Hamiltonian matrix. If the molecule's dipole moment is along one of the principal axes of inertia, the matrix will be block diagonalized by this transformation according to the remaining symmetry in the field and the blocks can be treated independently. For arbitrary orientation of the dipole moment in the inertial frame of the molecule the full matrix must be diagonalized. In any case, this process ensures that all states (eigenvalues and eigenvectors) obtained from a single matrix diagonalization do have the same symmetry, and, therefore, no real crossings between these states can occur. Therefore, by sorting the resulting levels by energy and assigning quantum number labels in the same order as for the field-free states of the same symmetry yields the correct adiabatic labels. These calculations are performed for a number of electric field strengths - typically in steps of $1 \mathrm{kV} / \mathrm{cm}$ from $0 \mathrm{kV} / \mathrm{cm}$ to $200 \mathrm{kV} / \mathrm{cm}$ - and the resulting energies, $W_{\text {Stark }}(E)$, are stored for later use in simulations using the libcoldmol program package [78].

\section{Monte Carlo Simulations of a Molecular Beam}

Simulations of the electrostatic beam deflection in our setup are performed with the home-built software package libcoldmol [78]. Trajectories for individual molecules in a given rotational quantum states are obtained from numerical integration of the $3 \mathrm{D}$ equations of motion using a Runge-Kutta algorithm. The initial phase space distribution of the molecular packet in the transverse spatial coordinates, $x$ and $y$, is described by the mean values and widths of circular uniform distributions. For the velocity coordinates, Gaussian distributions characterized by their mean values and full widths at half maximum are used. Also the initial time spread of the molecular beam, which corresponds to the opening time of the valve, is described by a Gaussian distribution centered around $t_{0}$. From the initial phase space distribution, the position of a molecule in phase space is randomly chosen. Then, it is propagated through the beamline, which includes all mechanical apertures of the experimental setup. From the electric field in the electrostatic deflector, which is calculated in two dimensions using finite element methods (Comsol Multiphysics 3.4), and the Stark energy (see Appendix A 1), the force acting on the molecule in the transverse directions is obtained: $\vec{F}=-\vec{\nabla} W_{\text {Stark }}(E)$. The electric field is taken to be constant along $z$. Finally, the deflected molecules are propagated through field-free space to the detection region. For each quantum state, $10^{5}$ trajectories are calculated yielding single-quantum state deflection profiles $I_{s}(y)$.

\section{Deflection Profiles}

The spatial deflection profile for an ensemble of molecules at a given rotational temperature, $I\left(y, T_{\text {rot }}\right)$, is calculated from the single-quantum-state deflection profiles, $I_{s}(y)$, as follows:

$$
I\left(y, T_{\text {rot }}\right)=\frac{1}{w} \sum_{s=1}^{N} w_{s}\left(T_{\text {rot }}\right) I_{s}(y)
$$

Here, $N$ is the number of quantum states included in the simulations and $w_{s}\left(T_{\text {rot }}\right)$ is the population weight for a given quantum state:

$$
w_{s}\left(T_{\text {rot }}\right)=g_{M} g_{\text {ns }} e^{\frac{W_{0}-W_{s}}{k T_{\text {rot }}}}
$$

with $W_{0}$ being the field-free potential energy of the ground state and $W_{s}$ the field-free energy of the current state; $g_{M}=1$ for $M=0$ and $g_{M}=2$ otherwise; $g_{\text {ns }}$ accounts for the nuclear spin statistical weight of the current state. For both, benzonitrile and iodobenzene, $g_{\mathrm{ns}}=5$ for $K_{a}$ even and $g_{\mathrm{ns}}=3$ otherwise. The normalization is given by $w=\sum_{s=1}^{N} w_{s}$.

In some cases, a molecular beam cannot be accurately described by a single rotational temperature [46]. Therefore, a two-temperature model is implemented in our simulations. In the two-temperature model, the lowtemperature part of the molecular beam is calculated as described above. In order to realistically simulate the high-temperature component in the molecular beam, a very large number of quantum states would have to be included in the simulations for large asymmetric top molecules. Because these Monte Carlo simulations are very time consuming, the high-temperature component is approximated by adding a small fraction of undeflected molecules to the deflected beam profile. This approximation is well justified, because typical rotational temperatures for the high-temperature component in a molecular beam are on the order of $10 \mathrm{~K}$ and, for these temperatures, most of the molecules reside in high rotational quantum states that have a small Stark shift and, therefore, remain almost undeflected. Thus, the deflection profile, $I_{2 \mathrm{~T}}\left(y, T_{\mathrm{rot}}, q\right)$, in the two-temperature model is calculated as

$$
I_{2 \mathrm{~T}}\left(y, T_{\mathrm{rot}}, q\right)=q \cdot I\left(y, T_{\mathrm{rot}}\right)+(1-q) \cdot I_{\mathrm{ud}}(y)
$$

where $0<q<1$ and $I_{\mathrm{ud}}(y)$ denotes the undeflected spatial beam profile that is obtained when both electrodes are grounded.

\section{Fit of Rotational Temperature}

In order to calculate the rotational temperature of the molecular beam, the simulated deflection profiles are fit- 
rotational temperature.

ted to the experimental data using a Nelder-Mead simplex algorithm. In the fitting procedure, the difference between simulated and measured deflection profiles is minimized, where the rotational temperature $T_{\text {rot }}$ of the low-temperature component, the fraction $q$ of molecules in this low-temperature component, and a general intensity scaling factor $s$ of the deflected profiles (with respect to the undeflected beam profile) are used as fitting parameters. All deflection curves measured for different high voltages are fitted simultaneously to determine the rotational temperature.

\section{Acknowledgments}

We thank Henrik Haak for expert technical support. This work is further supported by the Carlsberg Foundation, the Lundbeck Foundation, the Danish Natural Science Research Council, and the Deutsche Forschungsgemeinschaft within the priority program 1116.
[1] H. J. Loesch and A. Remscheid, J. Chem. Phys. 93, 4779 (1990).

[2] B. Friedrich and D. R. Herschbach, Nature 353, 412 (1991).

[3] B. Friedrich and D. Herschbach, Phys. Rev. Lett. 74, 4623 (1995).

[4] H. Stapelfeldt and T. Seideman, Rev. Mod. Phys. 75, 543 (2003).

[5] B. Friedrich and D. Herschbach, J. Chem. Phys. 111, 6157 (1999).

[6] B. Friedrich and D. Herschbach, J. Phys. Chem. A 103, 10280 (1999).

[7] F. Filsinger, U. Erlekam, G. von Helden, J. Küpper, and G. Meijer, Phys. Rev. Lett. 100, 133003 (2008).

[8] J. C. H. Spence and R. B. Doak, Phys. Rev. Lett. 92, 198102 (2004).

[9] E. R. Peterson, C. Buth, D. A. Arms, R. W. Dunford, E. P. Kanter, B. Krassig, E. C. Landahl, S. T. Pratt, R. Santra, S. H. Southworth, and L. Young, Astrophys. Lett. \& Comm. 92, 094106 (2008).

[10] J. Levesque, Y. Mairesse, N. Dudovich, H. Pépin, J.-C. Kieffer, P. B. Corkum, and D. M. Villeneuve, Phys. Rev. Lett. 99, 243001 (2007).

[11] J. Itatani, J. Levesque, D. Zeidler, H. Niikura, H. Pépin, J. C. Kieffer, P. B. Corkum, and D. M. Villeneuve, Nature 432, 867 (2004).

[12] S. Stolte, in Atomic and molecular beam methods, edited by G. Scoles (Oxford University Press, New York, NY, USA, 1988), vol. 1, chap. 25, pp. 631-652.

[13] H. Kallmann and F. Reiche, Z. Phys. 6, 352 (1921).

[14] E. Wrede, Z. Phys. 44, 261 (1927).

[15] O. Stern, Z. Phys. 39, 751 (1926).

[16] I. I. Rabi, S. Millman, P. Kusch, and J. R. Zacharias, Phys. Rev. 55, 526 (1939).

[17] H. G. Bennewitz and W. Paul, Z. Phys. 139, 489 (1954).

[18] H. G. Bennewitz, W. Paul, and C. Schlier, Z. Phys. 141, 6 (1955).

[19] J. P. Gordon, H. J. Zeiger, and C. H. Townes, Phys. Rev. 95, 282 (1954).

[20] J. P. Gordon, H. J. Zeiger, and C. H. Townes, Phys. Rev. 99, 1264 (1955).

[21] H. G. Bennewitz, K. H. Kramer, J. P. Toennies, and W. Paul, Z. Phys. 177, 84 (1964).

[22] P. R. Brooks and E. M. Jones, J. Chem. Phys. 45, 3449 (1966).

[23] R. J. Beuhler, R. B. Bernstein, and K. H. Kramer, J.
Am. Chem. Soc. 88, 5331 (1966).

[24] D. H. Parker and R. B. Bernstein, Annu. Rev. Phys. Chem. 40, 561 (1989).

[25] E. W. Kuipers, M. G. Tenner, A. Kleyn, and S. Stolte, Nature 334, 420 (1988).

[26] T. P. Rakitzis, A. J. van den Brom, and M. H. M. Janssen, Science 303, 1852 (2004).

[27] H. L. Bethlem, G. Berden, and G. Meijer, Phys. Rev. Lett. 83, 1558 (1999).

[28] R. Fulton, A. I. Bishop, and P. F. Barker, Phys. Rev. Lett. 93, 243004 (2004).

[29] N. Vanhaecke, U. Meier, M. Andrist, B. H. Meier, and F. Merkt, Phys. Rev. A 75, 031402(R) (2007).

[30] D. Auerbach, E. E. A. Bromberg, and L. Wharton, J. Chem. Phys. 45, 2160 (1966).

[31] H. L. Bethlem, M. R. Tarbutt, J. Küpper, D. Carty, K. Wohlfart, E. A. Hinds, and G. Meijer, J. Phys. B 39, R263 (2006).

[32] M. R. Tarbutt and E. A. Hinds, New J. Phys. 10, 073011 (2008).

[33] K. Wohlfart, F. Grätz, F. Filsinger, H. Haak, G. Meijer, and J. Küpper, Phys. Rev. A 77, 031404(R) (2008).

[34] R. Moro, X. Xu, S. Yin, and W. A. de Heer, Science 300, 1265 (2003).

[35] M. Broyer, R. Antoine, I. Compagnon, D. Rayane, and P. Dugourd, Phys. Scr. 76, C135 (2007).

[36] L. Holmegaard, J. H. Nielsen, I. Nevo, H. Stapelfeldt, F. Filsinger, J. Küpper, and G. Meijer, Phys. Rev. Lett. 102, 023001 (2009).

[37] M. Hillenkamp, S. Keinan, and U. Even, J. Chem. Phys. 118, 8699 (2003).

[38] V. Kumarappan, C. Z. Bisgaard, S. S. Viftrup, L. Holmegaard, and H. Stapelfeldt, J. Chem. Phys. 125, 194309 (2006).

[39] U. Buck and M. Fárník, Int. Rev. Phys. Chem. 25, 583 (2006).

[40] S. Minemoto, H. Nanjo, H. Tanji, T. Suzuki, and H. Sakai, J. Chem. Phys. 118, 4052 (2003).

[41] B. Friedrich and D. Herschbach, J. Phys. Chem. 99, 15686 (1995).

[42] H. Sakai, C. P. Safvan, J. J. Larsen, K. M. Hilligsøe, K. Hald, and H. Stapelfeldt, J. Chem. Phys. 110, 10235 (1999).

[43] U. Even, J. Jortner, D. Noy, N. Lavie, and N. CossartMagos, J. Chem. Phys. 112, 8068 (2000).

[44] N. F. Ramsey, Molecular Beams, The International Se- 
ries of Monographs on Physics (Oxford University Press, London, GB, 1956).

[45] K. Wohlfart, M. Schnell, J.-U. Grabow, and J. Küpper, J. Mol. Spec. 247, 119 (2008).

[46] Y. R. Wu and D. H. Levy, J. Chem. Phys. 91, 5278 (1989).

[47] G. Berden, W. L. Meerts, and E. Jalviste, J. Chem. Phys. 103, 9596 (1995).

[48] O. Dorosh, E. Białkowska-Jaworska, Z. Kisiel, and L. Pszczółkowski, J. Mol. Spec. 246, 228 (2007).

[49] K. Wohlfart, F. Filsinger, F. Grätz, J. Küpper, and G. Meijer, Phys. Rev. A 78, 033421 (2008).

[50] M. Kirste, B. G. Sartakov, M. Schnell, and G. Meijer, Phys. Rev. A 79, 051401(R) (2009).

[51] J. J. Larsen, H. Sakai, C. P. Safvan, I. Wendt-Larsen, and H. Stapelfeldt, J. Chem. Phys. 111, 7774 (1999).

[52] A. Gijsbertsen, W. Siu, M. F. Kling, P. Johnsson, P. Jansen, S. Stolte, and M. J. J. Vrakking, Phys. Rev. Lett. 99, 213003 (2007).

[53] O. Ghafur, A. Rouzee, A. Gijsbertsen, W. K. Siu, S. Stolte, and M. J. J. Vrakking, Nature Phys. 5, 289 (2009).

[54] C. Ng, Annu. Rev. Phys. Chem. 53, 101 (2002).

[55] W. Li, X. Zhou, R. Lock, S. Patchkovskii, A. Stolow, H. C. Kapteyn, and M. M. Murnane, Science 322, 1207 (2008).

[56] F. Rosca-Pruna and M. J. J. Vrakking, Phys. Rev. Lett. 87, 153902 (2001).

[57] T. Seideman, J. Chem. Phys. 115, 5965 (2001).

[58] M. Machholm, J. Chem. Phys. 115, 10724 (2001).

[59] F. Rosca-Pruna and M. J. J. Vrakking, J. Chem. Phys. 116, 6579 (2002).

[60] E. Péronne, M. D. Poulsen, H. Stapelfeldt, C. Z. Bisgaard, E. Hamilton, and T. Seideman, Phys. Rev. A 70, 063410 (2004)

[61] M. Muramatsu, M. Hita, S. Minemoto, and H. Sakai, Phys. Rev. A 79, 011403(R) (2009).

[62] J. J. Larsen, K. Hald, N. Bjerre, H. Stapelfeldt, and T. Seideman, Phys. Rev. Lett. 85, 2470 (2000).

[63] H. Tanji, S. Minemoto, and H. Sakai, Phys. Rev. A 72, $063401(2005)$

[64] K. F. Lee, D. M. Villeneuve, P. B. Corkum, A. Stolow, and J. G. Underwood, Phys. Rev. Lett. 97, 173001 (2006).

[65] S. S. Viftrup, V. Kumarappan, S. Trippel, H. Stapelfeldt, E. Hamilton, and T. Seideman, Phys. Rev. Lett. 99, 143602 (2007).

[66] A. Rouzee, S. Guerin, O. Faucher, and B. Lavorel, Phys. Rev. A 77, 043412 (2008).
[67] I. Nevo, L. Holmegaard, J. H. Nielsen, J. L. Hansen, H. Stapelfeldt, F. Filsinger, G. Meijer, and J. Küpper, Phys. Chem. Chem. Phys. (2009), accepted, preprint available at http://arxiv.org/abs/0906.2971.

[68] R. D. Suenram and F. J. Lovas, J. Am. Chem. Soc. 102, $7180(1980)$

[69] T. R. Rizzo, Y. D. Park, L. Peteanu, and D. H. Levy, J. Chem. Phys. 83, 4819 (1985)

[70] F. Filsinger, J. Küpper, G. Meijer, J. L. Hansen, J. Maurer, J. H. Nielsen, L. Holmegaard, and H. Stapelfeldt, Angew. Chem. Int. Ed. 48 (2009), accepted.

[71] C. Z. Bisgaard, O. J. Clarkin, G. Wu., A. M. D. Lee, O. Geßner, C. C. Hayden, and A. Stolow, Science 323, 1464 (2009).

[72] C. B. Madsen, L. B. Madsen, S. S. Viftrup, M. P. Johansson, T. B. Poulsen, L. Holmegaard, V. Kumarappan, K. Jørgensen, and H. Stapelfeldt, Phys. Rev. Lett. 102, 073007 (2009)

[73] J. K. G. Watson, in Vibrational Spectra and Structure, edited by J. R. Durig (Marcel Dekker, 1977), vol. 6, chap. 1.

[74] M. Abd El Rahim, R. Antoine, M. Broyer, D. Rayane, and P. Dugourd, J. Phys. Chem. A 109, 8507 (2005).

[75] J. K. G. Watson, Can. J. Phys 53, 2210 (1975).

[76] P. R. Bunker and P. Jensen, Molecular Symmetry and Spectroscopy (NRC Research Press, Ottawa, Ontario, Canada, 1998), 2nd ed.

[77] S. C. Wang, Phys. Rev. 34, 243 (1929).

[78] J. Küpper and F. Filsinger, libcoldmol: A particle trajectory calculation framework (2003-2008), URL: http: //libcoldmol.cold-molecules.info.

[79] On the other hand, the local effective dipole moments of the neighboring states (dashed lines in Figure $6 \mathrm{a}$ ) are reduced by these crossings. At an avoided crossing, the two levels that are involved "exchange" their effective dipole moments. For large asymmetric top molecules, many avoided crossings can lead to a complicated shape of the adiabatic Stark curve and a strongly varying effective dipole moment with the electric field strength.

[80] In principle, deflection of the quantum states that are coincidentally polar for the range of electric field strengths in the deflector could be reduced by operating the deflector at a different high voltage. However, due to the large number of quantum states populated and the resulting large number of avoided crossings, other quantum states might be conincidentally polar for these operation conditions.

[81] Note that in reference 74 representation $I^{l}$ is used. 